on base-pair interactions, but the energy differences between correct and incorrect pairings are not large enough to explain such high fidelity, therefore DNA polymerases must also be active in the rejection of incorrect nucleotides.

From crystal structures, DNA polymerases are known to adopt two different conformations. An open conformation allows either correct or incorrect nucleotides to bind, which are then thought to be delivered to a reaction site. At this point a closed 'reaction-ready' conformation is adopted and the enzyme can 'check' whether the nucleotide is appropriate - it is proposed that, through base-pair interactions, this conformation can only be fully stabilized by the right nucleotide.

Now, a team from The Scripps Research Institute led by David Millar have observed a third intermediate conformation that acts as a fidelity checkpoint, allowing the polymerase to reject an incorrect nucleotide before it adopts the closed conformation. The team used single-molecule Förster resonance energy transfer (smFRET) to monitor changes in conformation in Escherichia coli DNA polymerase I.

Three conformational states were observed, corresponding to the closed and open conformations, and a previously unobserved intermediate; the 'ajar' state. During the process of nucleotide selection, the closed conformation was mostly observed when the correct nucleotide was present. However, in the presence of the incorrect nucleotide, the ajar conformation was the most likely to be seen. These observations imply a mechanism by which the polymerase rapidly switches from the open to the active closed conformation when the correct nucleotide is present, but blocks such a progression when the incorrect nucleotide is present. Rather, it gets held up in the intermediate conformation and induces the dissociation of the mismatched nucleotide.

\section{DNA BINDING}

\section{Visible changes}

J. Am. Chem. Soc. 134, 10214-10221 (2012)

Metal complexes that combine photosensitivity with the ability to bind to DNA have attracted much attention in the biomedical field. The fact that their fluorescence changes in the presence of DNA may prove useful for sensing applications. Moreover, their DNAbinding ability - which, in turn, can affect biological processes - can be switched on or off in a controlled manner through light irradiation at specific locations. Using scanning force microscopy, a team of researchers in Belgium led by Moucheron, Kirsch-De Mesmaeker and De Feyter have now described the mechanical and structural effects of the binding and photoreaction of a ruthenium complex on long DNA duplexes.
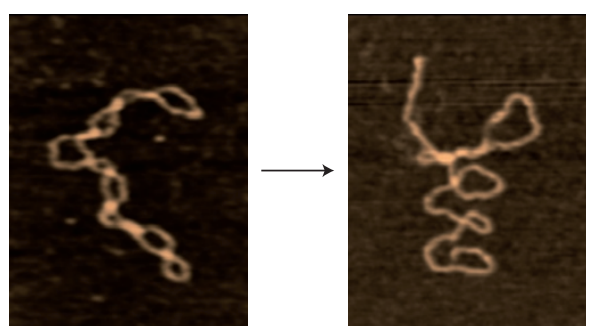

The ruthenium complex in question comprised two tetraaza-phenanthrene (TAP) moieties - phenanthroline (phen) derivatives in which two carbon atoms are substituted by nitrogen - and a third ligand, a phenanthrolino-hexaazatriphenylene polyaromatic group, known to intercalate in DNA by sliding between two base pairs. Comparisons between the behaviour of the TAP-based complex and its phen-based analogue, along with studies in the presence of strong hydrogenbond donor urea, suggest that - as well as the expected intercalation hydrogen bonding takes place between the uncoordinated nitrogen atoms of TAP and distant DNA segments. This effectively leads to intra- and inter-strand crosslinking, causing the DNA to fold and form large aggregates.

When irradiated, electronically excited TAP-based ruthenium complexes bound to DNA are known to undergo photoelectron transfer with neighbouring guanine residues. This process is followed by back electron transfer or the formation of a complex-duplex adduct, or - in the case of long strands of DNA - cleavage of the sugar-phosphate backbone. The Belgium-based team found that visiblelight irradiation of a supercoiled DNA strand (pictured left) in the presence of the TAP-based complex induced the cleavage of some single strands - which releases some structural strain - as well as the formation of adducts. These adducts can result in increased rigidity of the DNA and crosslinking between different segments. Such mechanical and topological changes may impact the biomedical applications of these ruthenium-TAP complexes.

Written by Gavin Armstrong, Stuart Cantrill, Stephen Davey and Anne Pichon.

\section{blog $_{\text {roll }}$ 㲾}

\section{Toxicity and death}

Bloggers breathe life into wounded chemicals and contemplate the death of organic synthesis.

For about a week, the chemical blogosphere became a toxic environment, but the only thing that bloggers sought to poison was public misperception. In a carnival spearheaded by Matt Hartings of American University, over 20 bloggers authored posts about their favourite toxic chemicals (http:// go.nature.com/dWTHzH). Hartings hosted the carnival on his site, ScienceGeist, in an effort to emphasize that many chemicals demonized in the media as 'toxic' have safe uses of immense practical value. "Chemicals aren't inherently good or bad," he writes, "in most cases, the danger is in the dosage."

Dr Rubidium, an analytical chemist who blogs at the Journal of Are You Fucking Kidding, contrasted several cases of homicide by the paralytic agent succinylcholine with its medical use in life-saving tracheal intubations (http:// go.nature.com/bFQFv6). Although that post was shockingly free of swear words, an ode to tetracyanoethylene (TCNE) on Carbon-Based Curiosities was as vulgar as it was informative (http://go.nature.com/ AmOzuB). Long-time blogger Excimer noted that "even the chemical industry is starting to shy away from chemicals" before proudly hailing applications of TCNE in the synthesis of the first organic ferromagnet and as a "highly efficient unicorn killer."

And speaking of death...debate over whether to sustain research in organic synthesis flared up again when Chemjobber sifted through a 120-page report on graduate education and found a rather provocative question inspired by Harvard chemist George Whitesides: "Should U.S. graduate students be doing organic synthesis if most organic synthesis is being done in China?" (http://go.nature.com/ uXbXMf). Derek Lowe at In the Pipeline prefaces his analysis with the statement, "If it hasn't crossed your mind, you haven't thought hard enough about the issues yet." (http://go.nature.com/fMbQuP).

Written by Paul Bracher, who blogs at http://blog.chembark.com 